\title{
Research on the Influence of Sodium Ion on Mechanism of Polymer Solution Viscosity Loss
}

\author{
Chun'an DU', Xianya ZHANG ${ }^{\mathrm{a}}$, Weiwei $\mathrm{CHEN}^{\mathrm{b}}$ and Peng XU' $\mathrm{X}^{\mathrm{c}, 1}$ \\ ${ }^{a}$ College of Engineering, China University of Petroleum-Beijing at Karamay, Karamay \\ 834000, China \\ b Deshi Energy Technology Group Co., Ltd, Dongying 257000, China \\ c Shengli Oil Field, SINOPEC, Dongying 257000, China
}

\begin{abstract}
The researches on the influence of sodium ion on mechanism of polymer solution viscosity loss were conducted. Scanning electron microscopy was used to analyze the polymer microstructure. Molecular dynamics simulation was employed to reveal the influence of sodium ion on the polymer molecular configuration. The results shown: the polymer viscosity loss was more than $70 \%$ when the concentration of sodium ion was above $4000 \mathrm{mg} / \mathrm{L}$. The results of microstructure and molecular conformation analysis indicated that the main reason of viscosity loss was the electrostatic attraction between sodium ion and negatively charged groups of polymer molecule chains, which cause compression of polymer molecular chain. The coil and shrinkage of polymer molecular chain led to the breakage of the spatial network structure of macromolecules.
\end{abstract}

Keywords. Sodium ion, polymer solution viscosity loss, polymer microstructure, molecular dynamics simulation

\section{Introduction}

At present, most oilfields in China have come into middle and late stage of waterflood development. Chemical flooding has become an important measure to enhance oil recovery. Oil resource potential evaluation showed that the recoverable oil reserves can be increased 1.18 billion tons through the EOR (Enhanced Oil Recovery) methods, such as polymer flooding, alkaline/polymer binary composite flooding and alkaline/surfactant/polymer (ASP) combinational flooding [1]. Polymer solution enhances oil recovery by increasing the viscosity and retention time of the injection fluid, reducing reservoir permeability to adjust the water absorption profile, and improving the oil flow ratio and actual conformability. Therefore, the viscosity of polymer solution is an important factor affecting the development effect of chemical flooding. Studies have found that polymer molecular structure, molecular weight size, degree of hydrolysis and mechanical shear, salt ions, temperature, and other factors all affect polymer viscosity, thus affecting the development effect of polymer flooding

${ }^{1}$ Corresponding Author, Peng XU, Shengli Oil Field, SINOPEC, Dongying 257000, China; Email: 2018592017@cupk.edu.cn. 
[2-5].

In recent years, a lot of researches have been done on the influence of different ions on viscosity of polymer solution. But most of them are concentrated in the influence on the apparent viscosity and the stability of the polymer solution. The researches on the inner mechanism of the polymer solution viscosity loss and the micro structure of the polymer are less [6-8]. The conformation of polymer chains in solution is closely related to the viscosity of the solution, so it is necessary to study the effect of different ions on the conformation of polymer chains.

In this paper, the scanning electron microscopy was used to observe the microscopic morphology of polymer solution before and after the action of the sodium ion, and the mechanism of the action of sodium ion on polymer molecular chain configuration was studied by molecular dynamics method. The internal mechanism of polymer viscosity loss caused by sodium ions was explored to provide technical support for improving the viscosity of polymer solution prepared by oilfield sewage and enhancing oil recovery.

\section{Materials and Methods}

\subsection{Experimental Materials and Instruments}

Polyacrylamide was produced by Shandong Polymer Biochemicals Co., Ltd., the hydrolysis degree is $25 \%$, the rest of the reagent is chemically pure.

Main instruments: Brookfield DV - III viscosity meter (Brookfield, USA); SU8020 Scanning Electron Microscope (Hitachi, Japan).

\subsection{Effect of Sodium Ion Concentration on Viscosity of Polymer Solution}

$5000 \mathrm{mg} / \mathrm{L}$ polymer mother liquor was prepared and then diluted with distilled water to $2000 \mathrm{mg} / \mathrm{L}$. different concentrations of sodium ions were added and incubated for $24 \mathrm{~h}$ at room temperature. The Brookfield DV - III was used to test the viscosity of the solution (0\# rotor, 6 RPM).

\subsection{Microscopic Morphology Analysis}

A small amount of the polymer solution described in 1.2.1 was taken and dispersed with $3 \mathrm{~mL}$ anhydrous ethanol for $3-5 \mathrm{~min}$ by ultrasound. Then drop it on the silicon wafer, and scanning electron microscope was used to observe its microscopic morphology.

\subsection{Molecular Dynamics Analysis}

All polymer solution system simulations employed ab initio calculations of the force field COMPASS. Model design was conducted by using the Construction tool in Amorphous Cell [9, 10]. 


\section{Results and Discussion}

\subsection{Effect of Different Ion Concentrations on the Viscosity of The Polymer Solutions}

The effect of different sodium ion concentration on the viscosity of polymer solutions is presented in figure 1.

As seen from the figure that with the increase of sodium ion concentration, the viscosity of polymer solution decreases rapidly at first and then becomes flat. When the sodium ion content exceeds $4000 \mathrm{mg} / \mathrm{L}$, the viscosity loss of the polymer is more than $70 \%$.

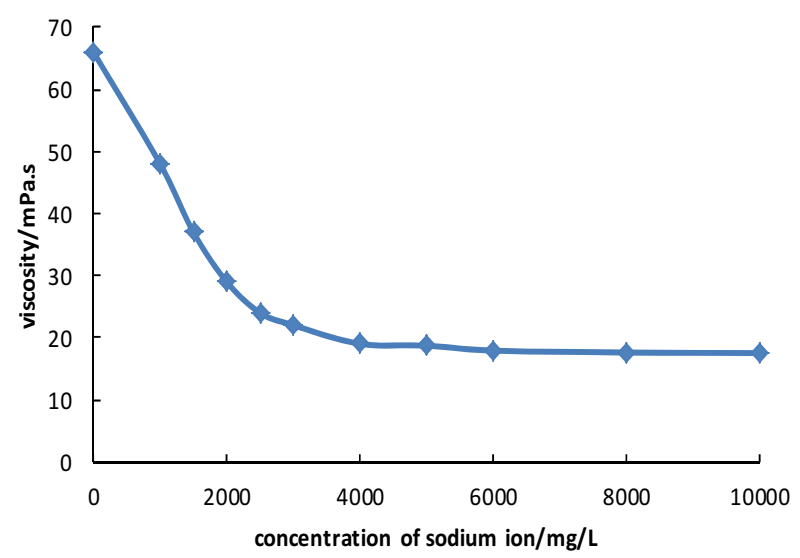

Figure 1. Effect of different sodium ion concentration on the viscosity of polymer solutions.

\subsection{Microscopic Morphology Analysis of the Polymer Solution}

The micro-morphology of polymer at different sodium ion concentrations is shown in figure 2. As concentration of sodium ion goes up, the extension degree of polyacrylamide molecular chain goes down. The polymer molecules gather together after shrinking, causing the three-dimensional network structures become thinner and loose. It is inferred that sodium ion compresses and diffuses the electric double layer, resulting in the gradual loosening of the molecular chain skeleton, the destruction of the three-dimensional network structure, and the decrease of viscosity.

\subsection{Molecular Dynamics Simulation}

\subsubsection{Model Construction}

The Construction tool in Amorphous Cell was used to build the models by dissolving the random HPAM molecular chain with hydrolysis degree of $25 \%$ in water. The specific parameters of the models are shown in table 1 . 


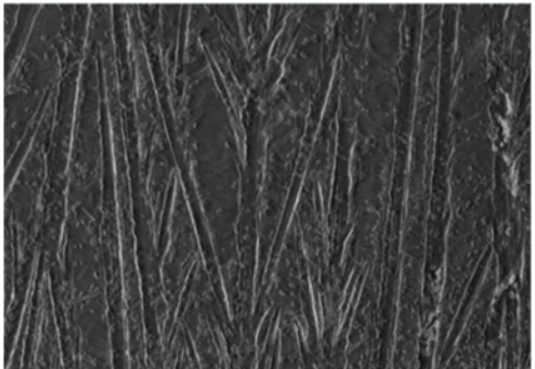

(a)

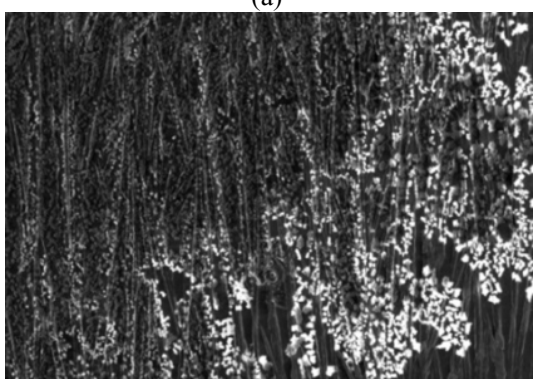

(c)

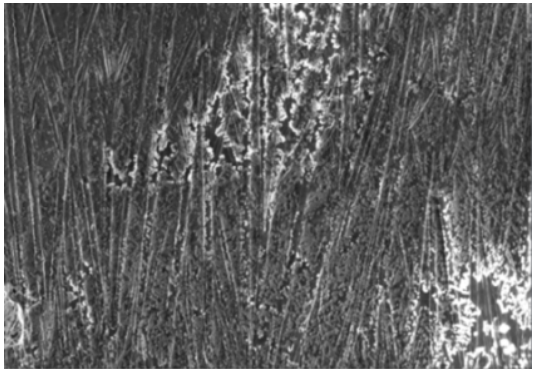

(b)

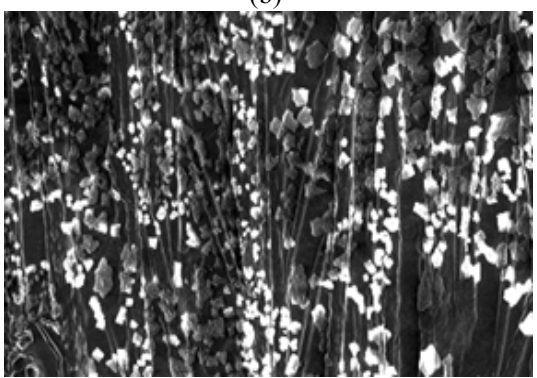

(d)

Figure 2. Microscopic morphology of polymer solution of different sodium ion concentrations: (a) blank; (b) $2000 \mathrm{mg} / \mathrm{L} \mathrm{Na}^{+}$; (c) $5000 \mathrm{mg} / \mathrm{L} \mathrm{Na}^{+}$; (d) $10000 \mathrm{mg} / \mathrm{L} \mathrm{Na}^{+}$.

Table 1. Model parameters of polymer solution with different sodium ions.

\begin{tabular}{llllll}
\hline Models & Cation & $\mathbf{N}_{\text {HPAM }}$ & $\mathbf{N}_{\text {Cation }}$ & $\mathbf{N}_{\text {H2O }}$ & $\mathbf{V} / \AA^{\mathbf{3}}$ \\
\hline a & $\mathrm{Na}^{+}$ & 1 & 5 & 2000 & $39.10^{3}$ \\
b & $\mathrm{Na}^{+}$ & 1 & 10 & 2000 & $39.10^{3}$ \\
c & $\mathrm{Na}^{+}$ & 1 & 20 & 2000 & $39.10^{3}$ \\
d & $\mathrm{Na}^{+}$ & 1 & 35 & 2000 & $39.10^{3}$ \\
\hline
\end{tabular}

\subsubsection{Molecular Configuration}

Figure 3 shows the microcosmic structures of polymer solution with different $\mathrm{NaCl}$ concentration. It can be seen that the polyacrylamide molecular chain extended better in the $\mathrm{NaCl}$-free system (figure $3 \mathrm{a}$ ). The polymer chains in the system with $\mathrm{NaCl}$ all shrink. The shrinkage of polymer chains increases as the $\mathrm{NaCl}$ concentration increase. With the increase of $\mathrm{NaCl}$ concentration, the shrinkage of polymer molecular chain can be divided into two steps. At first, the middle part of polymer chain tends to be close to each other, and then the both ends of polymer molecular chain approach to the middle part.

\subsubsection{Mean Square Radius of Gyration}

The radius of gyration and the end-to-end distance of the polymers were extracted from the above four systems to quantitatively describe the configuration changes of the polymers. Figure 4 shows the radius of gyration and end-to-end distance of polymer molecular chain under different concentrations of $\mathrm{NaCl}$. When the number of $\mathrm{NaCl}$ molecules added is within 20, the radius of gyration and the end-to-end distance of polymer chain decreases linearly. When the number is more than 20 , the radius of 
gyration of polymer chain decreases slightly, but end-to-end distance decreases greatly, which indicates that the polymer segments shrink greatly at this time, but the two ends of the polymer molecular chain are still close to each other. When the $\mathrm{NaCl}$ concentration is high enough, the polymer molecular chain shrinks, and the interweaving between molecular chains weakens, the viscosity of polymer solution decreases.
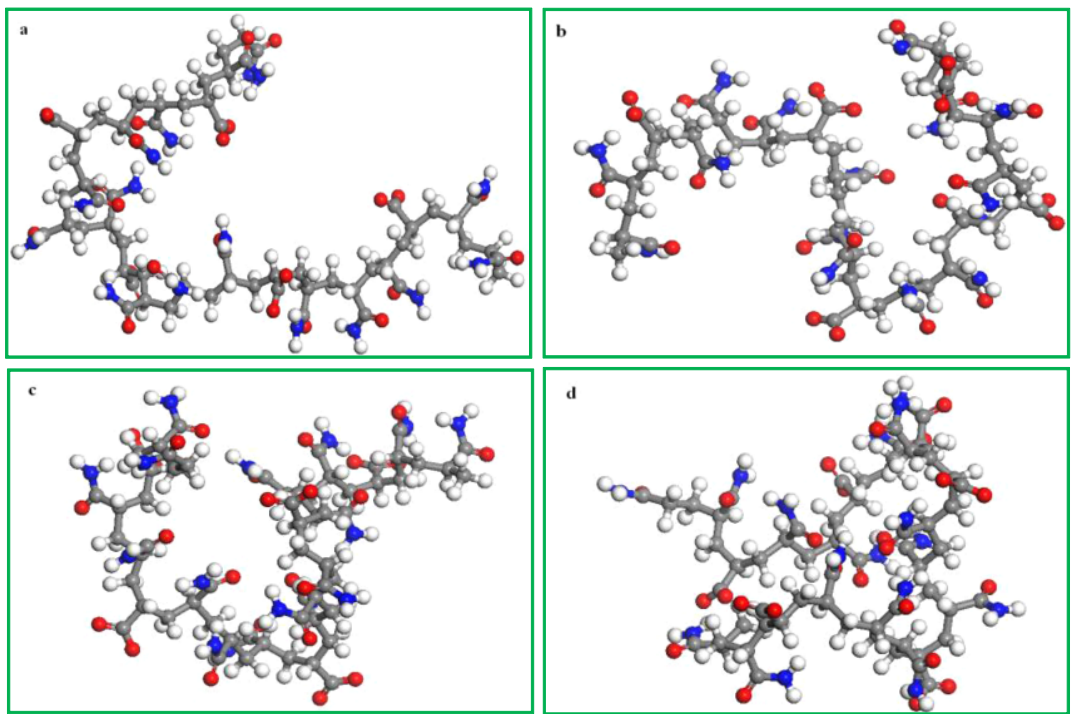

Figure 3. Configuration of polymer chain with different concentration of $\mathrm{NaCl}$ : (a) blank; (b) $10 \mathrm{Na}^{+}$; (c) 20 $\mathrm{Na}^{+} ;$(d) $35 \mathrm{Na}^{+}$.

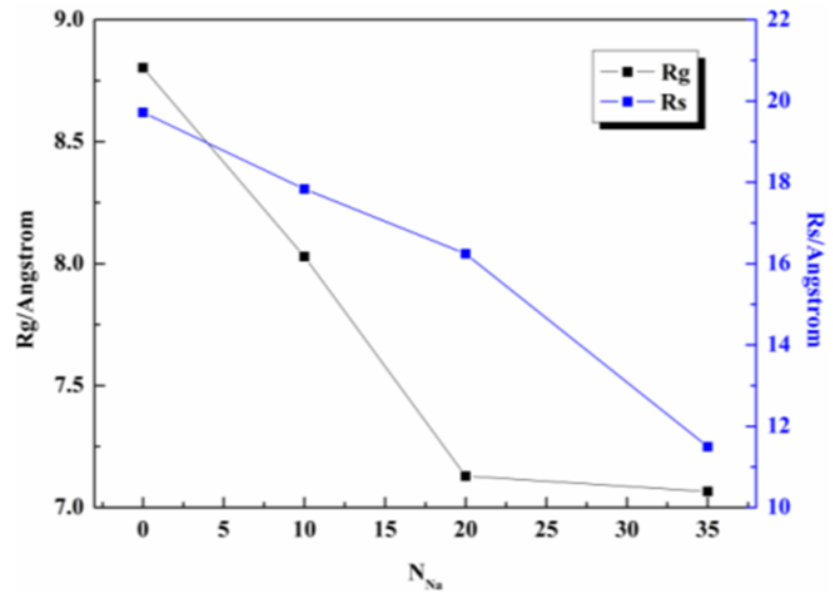

Figure 4. Radius of gyration and end-to-end distance of polymer chains with different concentration of $\mathrm{NaCl}$.

\subsubsection{Radial Distribution Function}

The radial distribution functions of different atoms were extracted and analyzed to explore the mechanism of the influence of sodium ion on polymer molecular chain 
from the structure of polymer molecular chain, water molecule and sodium ion.

Figure 5 shows the radial distribution functions between negatively charged carboxylic acid groups in polymer chains and oxygen atoms in water molecules with different $\mathrm{NaCl}$ concentrations. The position of the first peak in the four systems is 2.52 $\AA$. With the increase of $\mathrm{NaCl}$ concentration, the peak value gradually decreases, but each one is greater than $1 \AA$. The carboxylic acid groups in polymer molecular chains are negatively charged and have strong polarity, causing a strong interaction between the groups and water molecules with the same polarity. Therefore, the structural tightness of water molecules around carboxylic acid groups is greater than that of bulk phase water molecules. What can be seen from the decrease of the first peak value of the radial distribution function between polymer and water molecules is the effect of water molecules on polymer is gradually weakened. The value does not decrease obviously after adding $10 \mathrm{Na}^{+}$, which indicates that the effect of solvent water on the polymer molecular chains is still very strong when the $\mathrm{NaCl}$ concentration is small. When $20 \mathrm{NaCl}$ was added into the system, the first peak value decreased significantly, and the interaction between polymer molecular chains and water molecules was obviously weakened. The decrease becomes smaller after adding $35 \mathrm{NaCl}$, which indicates that when the amount of $\mathrm{NaCl}$ is more than 20 , the influence of solvent water molecules on the polymer tends to be stable.

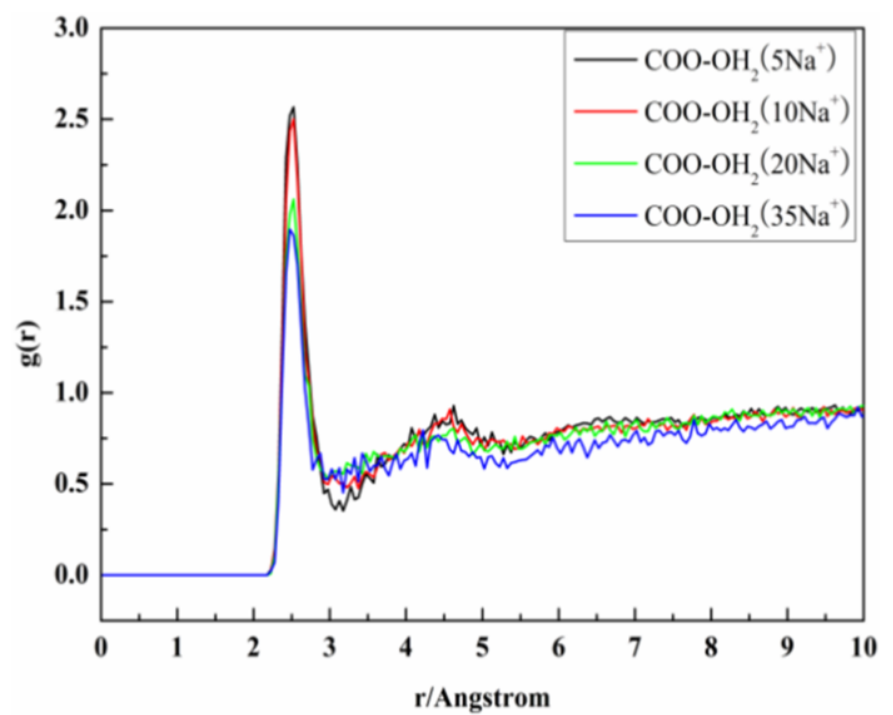

Figure 5. Radial distribution function between carboxylate groups and water molecules in polymer molecular chains with different amount of $\mathrm{NaCl}$.

In order to further explore the role of sodium concentration in the above process, the radial distribution function between carboxylate groups and sodium ion in polymer molecular chains was extracted (figure 6). When the concentration of sodium ion increases, the high concentration of anti-ion will form a layered ion layer around the polymer chain, which compresses the polymer chain, resulting in the shrinkage of the chain. Figure 6 shows that the first peak of the radial distribution function between carboxylate groups and sodium ion in three different sodium ion addition systems appears at $2.02 \AA$, which is closer than that between carboxylate groups and water 
molecules. Sodium ion is closer to the polymer than the water molecule around the chain, because the electrostatic interaction between carboxylate group and sodium ion is stronger than that between carboxylate group and water molecule. Since sodium ions are closer to the polymer chain and form an anti-ion layer, on the one hand, mutual exclusion between carboxyl acid groups within the polymer chain is shielded, on the other hand, the interaction between the polymer chain and water molecules is also shielded, resulting in the shrinkage of polymer chain segments. The first peak values of the radial distribution functions of carboxylic groups and sodium ions are $97.53 \AA$ and $97.11 \AA$ in the system containing 10 and 20 sodium ions, respectively, and $46.7 \AA$ in the system containing 35 sodium ions. When the amount of sodium ion is less than 20 , most of the sodium ions are very close to the polymer molecular chain. When the amount is more than 20, some sodium ions are dispersed in the bulk phase aqueous solution, and the effect of this part on the polymer becomes very little. In this paper, from the experimental part, when the inorganic salt concentration reaches a certain value, the viscosity of polymer solution tends to be stable and no longer decreases.

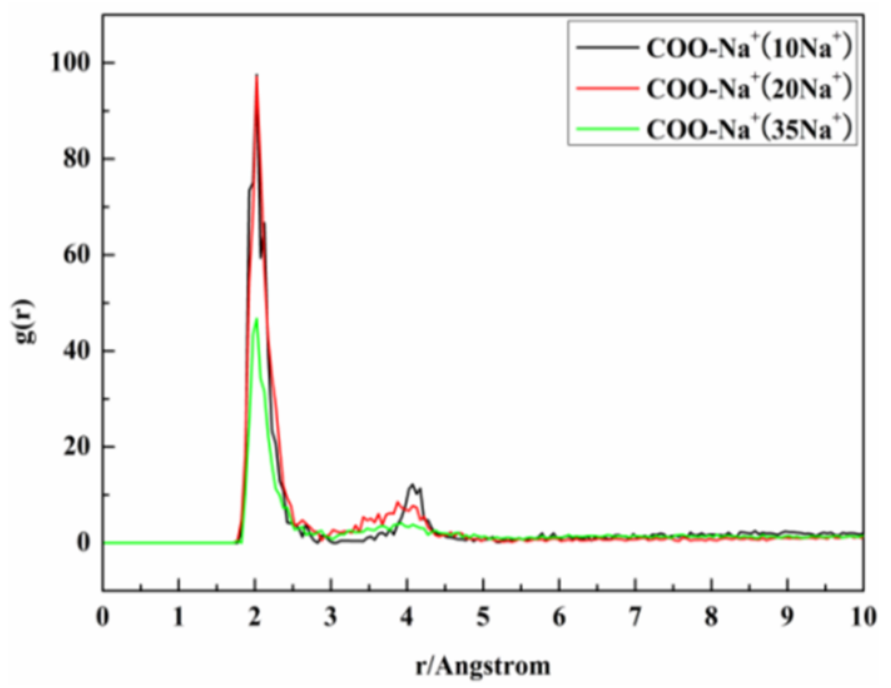

Figure 6. Radial distribution function between carboxylate group and sodium ion in polymer molecular chain with different amount of $\mathrm{NaCl}$.

\subsubsection{Interaction Energy}

The influence mechanism of different $\mathrm{NaCl}$ concentrations on the shrinkage behavior of polymer molecular chains was analyzed from the point of interaction energy. COMPASS force field is used to control the motion and potential energy of polymer and solvent molecules. The total energy can be divided into three categories: bond interaction energy of polymer, group cross interaction energy and non-bond interaction energy in polymer (including van der Waals interaction and Coulomb interaction). The calculation formula of the interaction energy between polymer and water molecules in the system is: $E_{\text {interaction }}=E_{\text {total }}-\left(E_{\text {water }}+E_{\text {polymer }}\right)$. Figure 7 shows the interaction energy at different $\mathrm{NaCl}$ concentrations. In the system with only 5 sodium ions, the interaction energy between polymer molecule chain and water molecule is the highest, and with the increase of $\mathrm{NaCl}$ concentration, the interaction energy decreases. The cause of this 
phenomenon is as follows: the sodium ion is close to the polymer molecular chain under the electrostatic action, and forms a sodium ion layer, which hinders the interaction between the polymer and water molecules. When more $\mathrm{NaCl}$ is added, the sodium ion layer around the polymer molecular chain is thickened, and the blocking effect is further strengthened. It can be seen from the change of interaction energy that the interaction energy between polymer molecular chain and water molecule decrease rapidly when sodium ion is lower than 20 . The low concentration of sodium ions close to polymer molecular chain and form ion layer due to electrostatic interaction, weakening the effect of water molecules on polymer molecular chain. When the sodium ion is higher than 20, the decreased magnitude of interaction energy lessens, because too much sodium ion does not enter into the ion layer around polymer molecular chain, but freezes in water, so these excess sodium ions do not weaken the interaction energy between polymer molecular chain and water molecule.

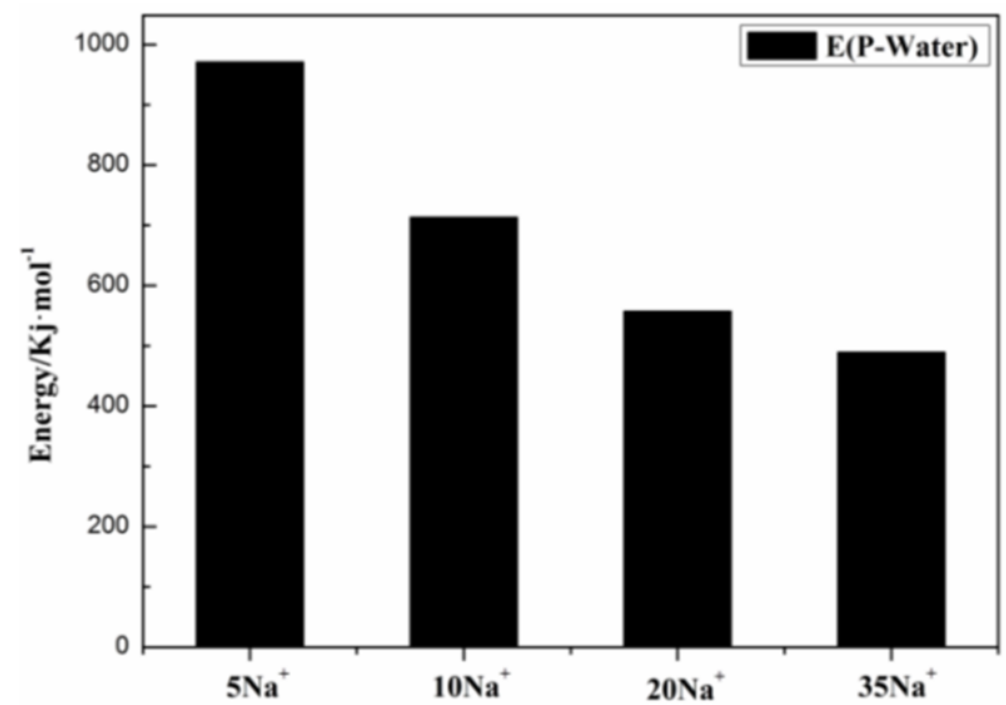

Figure 7. Interaction energy between polymer molecular chain and solvent water with different amount of $\mathrm{NaCl}$.

\subsubsection{Polymer Configuration Energy.}

Figure 8 shows the configuration energy of polymer molecular chain in the four systems. The larger the value is, the more stable and shrinking the configuration is. When 5 sodium ions are added into the system, the configuration energy of polymer molecular chain is the highest, and the configuration is the most extended. When the addition of $\mathrm{NaCl}$ increases, the inorganic salts shield the rejection of carboxylate ions in polymer molecular chains the effect of water molecules on the polymer molecular chains, resulting in the shrinkage of the polymer molecular chain so the configuration energy decreases. 


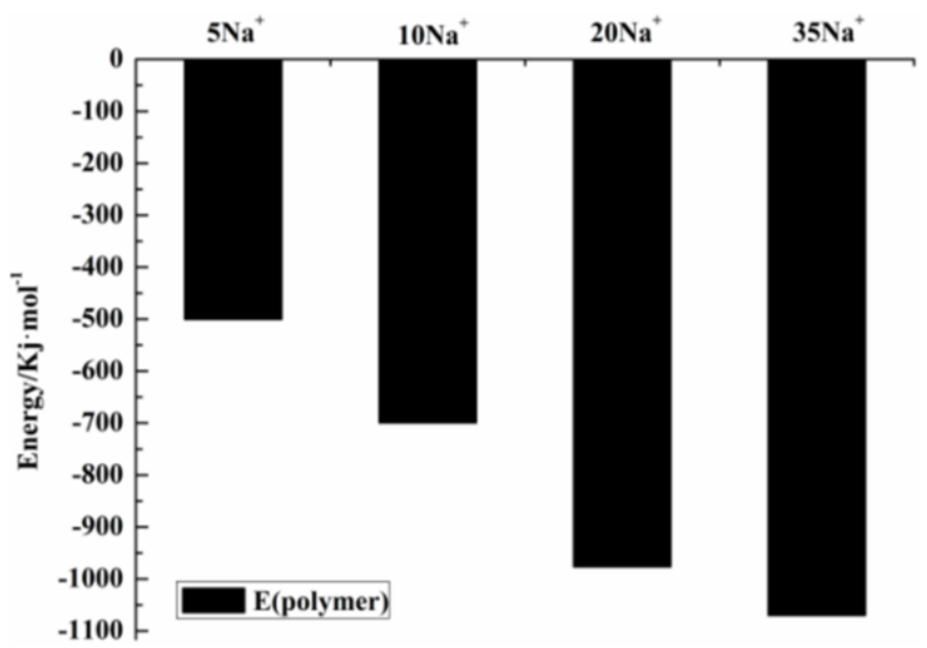

Figure 8. Configuration energy of polymer molecular chain with different $\mathrm{NaCl}$.

\section{Conclusion}

(1) The viscosity of polymer solution decreases with the increase of sodium ion concentration. When the concentration of sodium ion is higher than $4000 \mathrm{mg} / \mathrm{L}$, the viscosity loss is more than $70 \%$.

(2) The results of micro-morphology and molecular configuration analysis show that a strong electrostatic interaction between sodium ion and the charged carboxyl groups in the polymer molecular chain, which compresses the chain and destroys the space network structure of the polymer, and then decreases the viscosity of the polymer solution.

\section{References}

[1] Caulfield M J, Qiao G G and Solomon D H 2002 Chem. Rev. 1023067.

[2] Feng S D, Yuan D D, Sui X, et al. 2013 Appl. Mech. Mater. 268442.

[3] Liu J F, Feng J Y, Hu H, et al. 2018 J. Petrol. Sci. Eng. 170738.

[4] Choi J, Ka D, Chung T, et al. 2015 Macromol Res. 23518.

[5] Rashidi M, Blokhus A M and Skauge A 2011 J. Appl. Polym. Sci. 1193623.

[6] Gao C 2013 J Petrol. Exp. Prot. 3203.

[7] Kang W L, Zhou Y, Wang Z W, et al. 2008 J. Cent. South Univ. T 15115.

[8] Song W, Zhang Y, Gao Y, et al. 2017 Chemosphere 189277.

[9] Du C A, Pan Y Q, Xu P, et al. 2016 J. China Univ. Petrol.: Nat. Sci. Ed. 40166.

[10] Pu Y, Zou Q, Hou D, Zhang Y and Chen S 2017 Carbohydr. Polym 15671. 